\title{
A CONCEPT FOR HIGH PERFORMANCE REFLECTOR-BASED SYNTHETIC APERTURE RADAR
}

\author{
Marwan Younis, Anton Patyuchenko, Sigurd Huber, Gerhard Krieger, and Alberto Moreira
}

German Aerospace Center (DLR), Microwaves and Radar Institute

\begin{abstract}
The success of current spaceborne Synthetic Aperture Radar (SAR) is boosting the performance requirement of next generation systems. In order to cope with the evolution of SAR the design of the new systems will need to meet higher requirements for spatial and radiometric resolution together with an increased availability. This tendency is recognized nearly independently of the application area and manifests itself through several study programs initiated by space agencies aiming at the design of future SAR systems. In this context the use of large reflectors combined with digital feed arrays for SAR is considered a possible alternative to planar array antennas. This paper suggests an X-band spaceborne SAR system utilizing a deployable reflector together with a digital feed array, analyzes its performance and highlights its advantages compared to other systems based on direct radiating arrays.
\end{abstract}

\section{INTRODUCTION}

A review of several ongoing studies for the conception of next generation SAR systems, reveals the shared characteristic of being multi-channel systems utilizing digital beamforming (DBF) techniques. Examples are the High Resolution Wide Swath (HRWS) SAR [1, 2, 3] and Tandem-L/DESDnyI mission [4, 5]. The common purpose of using multi-channel systems is to simultaneously obtain high spatial resolution and wide swath.

For DESDnyI/Tandem-L a reflector-based SAR system was first suggested, which was later extended to a hybrid architecture through a digital feed [6]. With this system it would be possible to image a swath width of $300-400 \mathrm{~km}$ [4]. Such a hybrid architecture has the potential to combine both the flexibility and the capabilities of DBF with the high antenna gain provided by a large reflector aperture. To lower the stowed satellite volume and weight, and therefore the launch costs, the reflector could be deployable. Unfurlable reflector antennas are a mature technology with extensive flight heritage in space telecommunications; satellites with lightweight mesh reflectors spanning diameters of $>20 \mathrm{~m}$ are already deployed in space [7].

From the above it seems reasonable to consider reflectorbased SAR systems for the future. In [8] a planar and a reflector system were designed to a common set of performance parameters; the comparison revealed that the reflector system can be realized with a simpler hardware and shows a performance advantage of several $\mathrm{dBs}$ in terms of ambiguity and signal-to-noise ratio.
The paper addresses this issue by suggesting a SAR system utilizing a reflector in conjunction with a digital feed array. Keeping future follow-up systems for the German TerraSAR-X and TanDEM-X SAR satellites in mind, the reflector system will be designed for X-band operation with performance requirement possibly exceeding those of HRWS [1]. In this paper emphasis will be given to the various operation modes and the performance; the antenna design is detailed in [9], while [10] elaborates on the performance improvement using dedicated DBF techniques and [11] addresses the issue of imaging gap removal by varying the pulse repetition frequency $(P R F)$.

\section{ARCHITECTURE AND OPERATION}

In 1981 Blyth [12] suggested a basic approach for analog beamsteering for a reflector system such that the receive beam moves over the swath in accordance with the direction of reflection. About twenty years later, his idea finds a more detailed description and justification in two independent and almost contemporary works $[13,14]$. Digital beamforming techniques in elevation and azimuth for a reflector are presented for the first time in [6].

In the following the digital beamforming technique and the corresponding system architecture is addressed. For clarity this will be given separately for the elevation and azimuth directions.

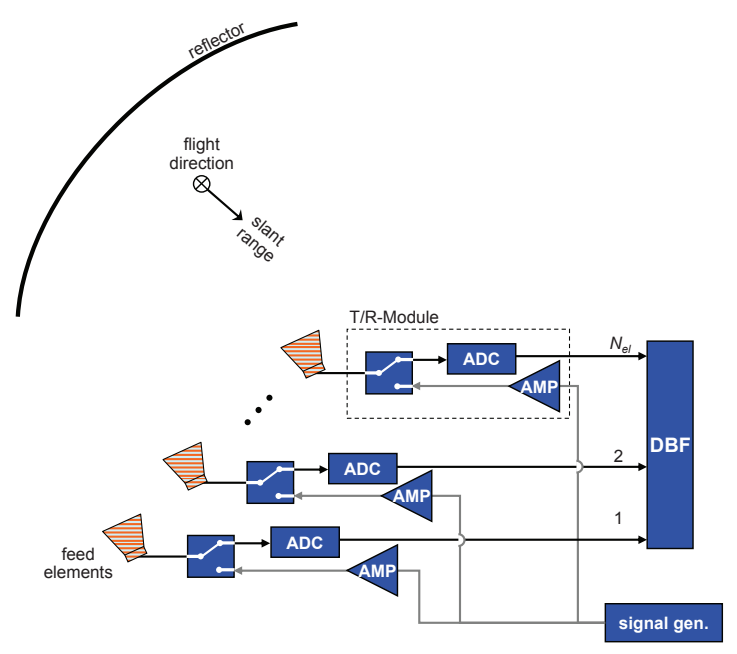

Fig. 1. System architecture for reflector system; shown here for a single azimuth but multiple elevation channels. 


\subsection{Digital Beamforming in Elevation}

The system (in elevation) consists of a parabolic reflector and a feed array of $N_{e l}$ antenna elements fed through transmit/receive modules, where an analog-to-digital converter (ADC) is placed after each T/R-module as shown in Fig. 1.

The SCan-On-REceive (SCORE) mode of operation [14], which is also suggested here, is primarily based on generating a wide transmit beam that illuminates the complete swath and a narrow, high gain receive beam that follows the pulse echo on the ground. SCORE results in an increased signal-to-noise ratio compensating the low gain of the transmit antenna and suppressing range ambiguities.

Activating all elements on transmit will generate a narrow beam illuminating a small portion of the reflector and by this gives a wide low gain beam illuminating the complete swath. On receive, the energy returned from a narrow portion of the ground illuminates the entire reflector and is focused on individual elements of the feed aperture. Each radar pulse traversing the swath causes the focused energy to sweep through all the feed elements within the time period of one pulse repetition interval $1 / P R F$ reduced by the transmit pulse duration. For a high $P R F$ multiple portions of the swath are illuminated instantaneously but each will activate a different subset of feed elements due to the different angle-of-arrival. Here, DBF consists of a complex summation of one or more subsets of the feed elements [10].

\subsection{Digital Beamforming in Azimuth}

A reflector system of multiple azimuth phase centers will require multiple feeds separated in the along track direction as shown in Fig. 2. Here each azimuth element "looks" at a different angle and by this covers a distinct angular (Doppler) segment. Thus each element samples a narrow Doppler spectrum corresponding to the half-power-beamwidth of the corresponding pattern. The PRF must be high enough such that the spatial sampling for each channel is adequate. This is approximately given by $P R F>2 \cdot V / D$ with the platform velocity $V$ and the reflector diameter $D$. If the Doppler spectra of the elements are contiguous, they jointly yield a higher azimuth resolution $\approx D /\left(2 N_{a z}\right)$. Each of the $N_{a z}$ Rx channels carries non-redundant information.

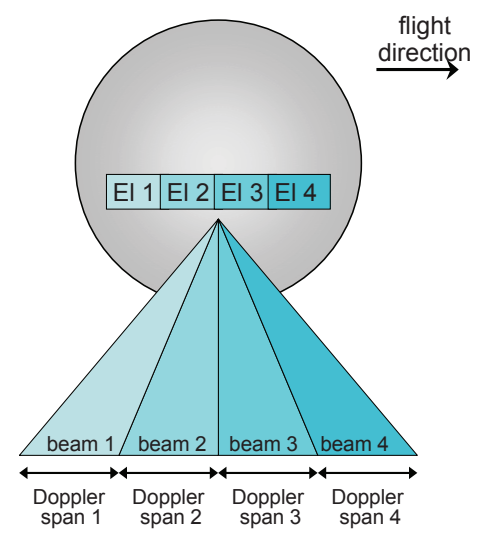

Fig. 2. Concept of digital beamforming in azimuth.

\section{SYSTEM DESIGN AND REQUIREMENTS}

The system is not designed for a specific set of user requirements which causes some difficulty in quantifying the SAR performance requirements. To overcome this difficulty we state the requirements based on state-of-the-art SAR systems. The later analysis will then give more insight into the actual performance and the possible compromises. The requirements are a resolution of $\approx 1 \times 1 \mathrm{~m}$, an ambiguity-to-signal ratio of $-20 \mathrm{~dB}$, and a noise-equivalent sigma zero of $-20 \mathrm{~dB}$. We assume an available average power of $2 \mathrm{~kW}$ which matches the value for the HRWS system [1].

\subsection{Orbit Selection}

The orbit selection is closely related to the intended application area and plays a crucial role for the reflector and feed design. Increasing the orbit height allows the reduction of the incidence angle range for the same swath width. It is noted that for direct radiating arrays increasing the orbit height is disadvantageous because of the degraded $S N R$ or increased antenna size; in the case of a reflector system, effective antenna areas in the order of $15-40 \mathrm{~m}^{2}$ can be realized, which makes higher orbits an attractive option since it releases the elevation scanning requirements.

For the mixed scientific and commercial application considered here the access range should be such that any arbitrary region on the Earth can be imaged at least once during the repeat cycle, however, global coverage within one repeat cycle is even preferable. The table below shows the access range and repeat cycle time for valid orbit configurations for orbit heights in the order of $750 \mathrm{~km}$. Global coverage within one repeat cycle is possible if the contiguous imaged swath of a single pass is equal to the access range given in the table. The reflector system is designed to image any sub-swath within the access range for any configuration. The instantaneous imaged swath will be $310 \mathrm{~km}$ thus allowing a global coverage within $\geq 9$ days.

\begin{tabular}{|l|ccccc|}
\hline \hline repeat cycle day & 7 & 8 & 9 & 11 & 12 \\
\hline access range $\mathrm{km}$ & 397 & 349 & 309 & 252 & 232 \\
\hline \hline
\end{tabular}

\subsection{Reflector and Digital Feed}

The antenna and feed design is elaborated in [9]. Here the description is restricted to reporting the values of the system used for the performance analysis. A circular rim reflector of diameter $\mathrm{D}=6 \mathrm{~m}$, focal length $\mathrm{F}=6.2 \mathrm{~m}$, and vertical (elevation) feed offset of $0.82 \mathrm{~m}$ is designed. The feed array consists of $4 \mathrm{x}$ 36 digital channels in azimuth and elevation, respectively. Each channel (connected to an ADC) may consist of one or more radiators.

\section{MODES OF OPERATION}

The digital feed allows operation in various modes which basically differ in the number of imaged sub-swathes and the way 
they are combined into one larger swath. These modes can be divided into four categories, where Fig. 3 shows an example timing diagram of each category:

- Single Stripmap This mode is well known from conventional SAR, where any sub-swath within the access range is imaged with a single burst and constant $P R F$. As mentioned in section 2.2 the azimuth processing needs to be adapted to the fact that each channel samples a narrow Doppler spectrum which are combined during processing.

- Multi Stripmap Here multiple sub-swathes of the same $P R F$ are imaged simultaneously allowing an increase of the total swath up to the access range. However, the imaged swath contains gaps (c.f. Fig. 3(b)) caused by the transmit instances. The gaps width is proportional to the pulse duty cycle. This mode takes advantage of DBF, since several SCORE beams are generated each one following the receive echo within one sub-swath.

- ScanSAR In this mode multiple bursts are used to increase the swath width. For the system shown here, a total of six to seven bursts would be required to cover the complete access range. An alternative would be to use ScanSAR to fill the gaps of the Multi-Stripmap mode; this would allow operation with only two bursts to image the complete access range. In any case the ScanSAR requires an adaption of the azimuth processing, which can become a challenge.

- PRF Variation Multiple sub-swathes are imaged at the same time but in addition the $P R F$ is varied from pulse to pulse. By this the gaps of the Multi-Stripmap mode can be avoided. This mode offers a highly attractive way to image an ultra wide swath but requires innovative processing approaches [11].

\section{SAR PERFORMANCE}

In the following the performance of the Multi-Stripmap mode is shown, since it is the most attractive one. The performance of the Single-Stripmap is identical to that of any single sub-swath. For the impact of the PRF-Veriation we refer to the separate investigation in [11].

The range performance given in terms of the range-ambiguityto-signal ratio $(R A S R)$ is shown in Fig. 4. Note that the echo signals from all the sub-swathes arrive simultaneously, something that typically causes range ambiguities in a conventional SAR (see Fig. 3(b)), here the narrow Rx SCORE pattern allows adequate range ambiguity suppression. The $R A S R$ is below $-40 \mathrm{~dB}$ which is possible because of the narrow low sidelobe SCORE Rx patterns shown exemplarily in Fig. 4(b).

The azimuth performance is given in terms of the azimuthambiguity-to-signal ratio ( $A A S R$ ) shown in Fig. 5. The $A A S R$ suffers from the degradation of the azimuth patterns at the nearrange boundary of the access range. This is basically an antenna

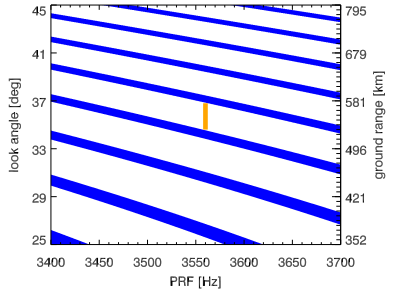

(a) Single Stripmap

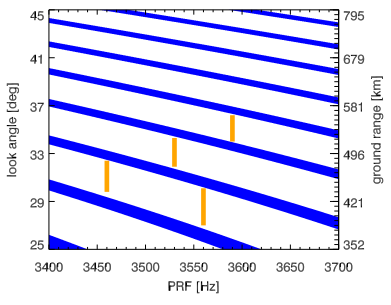

(c) ScanSAR

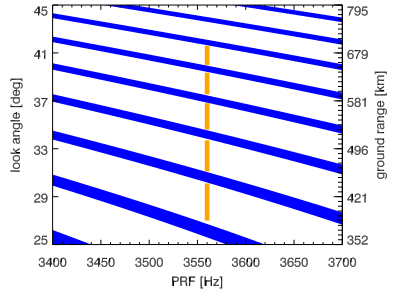

(b) Multi Stripmap

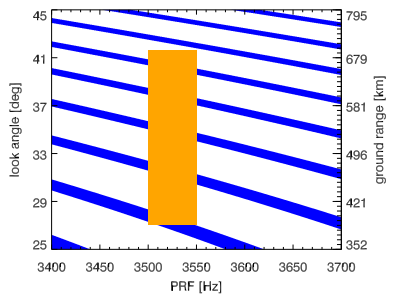

(d) $P R F$ Variation
Fig. 3. The timing diagram for different operation modes for a $745 \mathrm{~km}$ orbit and a pulse duty cycle of $10 \%$.

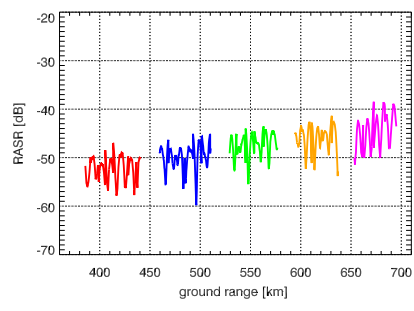

(a) $R A S R$

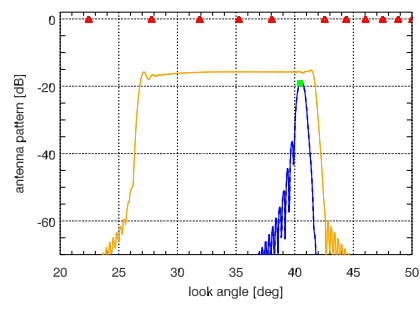

(b) elevation pattern
Fig. 4. Elevation ambiguity performance of reflector system.

and feed dimensioning issue which involves a compromise between the allowable maximum size and the performance. Here the $A A S R$ computed for a single azimuth channel is representative for the overall $A A S R$ as explained in section 2.2.

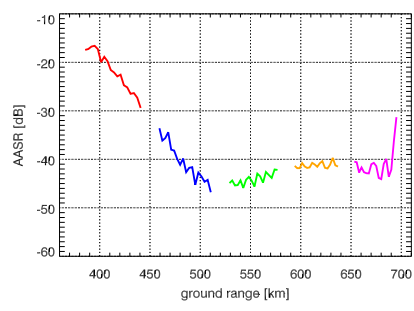

(a) $A A S R$

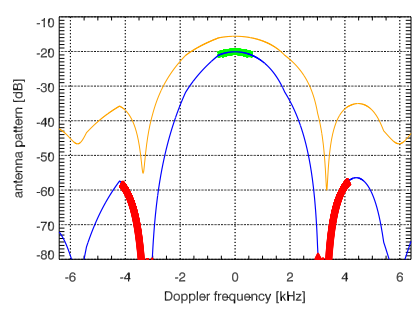

(b) azimuth pattern versus Doppler
Fig. 5. Azimuth ambiguity performance of reflector system.

The noise-equivalent sigma-zero for a total average transmit power of $2 \mathrm{~kW}$, a 2 -way system loss of $3 \mathrm{~dB}$, and a system noise temperature of $460 \mathrm{~K}$ is shown in Fig. 6(a). One characteristic of the reflector system with a transmit feed connected to T/R-modules (TRM) is that the power density on the ground does not decrease when illuminating a wider swath. As such the $N E S Z$ shown in Fig. 6(a) is valid independently of the number 
of imaged sub-swathes, however the average power consumption increases with the number of imaged sub-swathes.

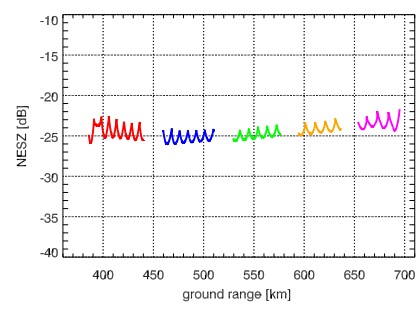

(a) $N E S Z$

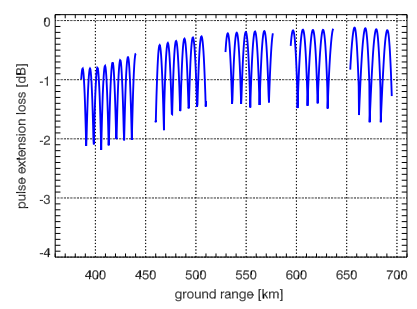

(b) pulse extension loss $(P E L)$
Fig. 6. Noise-equivalent sigma-zero and pulse extension loss of the reflector system.

In Fig. 6(b) the pulse extension loss $(P E L)$ is shown. This performance parameter describes the loss due to the nonvanishing pulse extension on the ground which is attenuated by the narrow RX SCORE antenna pattern. For the $10 \%$ duty cycle this loss is below $2 \mathrm{~dB}$.

\section{CONCLUSION}

Spaceborne SAR systems utilizing reflector antennas offer the possibility to improve the SAR performance. This performance improvement manifests itself through an increased swath width and a higher signal-to-noise ratio. The digital feed of a reflector system uses a smaller number of T/R-modules and by this require a higher average power per $\mathrm{T} / \mathrm{R}$-modules to radiate the same total power. Further the imaging modes shows a high potential for systems operating at low pulse duty cycle which require a higher peak power. The power requirements are not fulfilled with current T/R-modules at $\mathrm{X}$-band and thus require future technology development; here GaN technology seems to be a promising candidate. On-going research on advanced digital beamforming techniques for reflector systems show a high potential and should be continued.

\section{REFERENCES}

[1] C. Fischer, C. Schaefer, and C. Heer, "Technology development for the HRWS (High Resolution Wide Swath) SAR," in International Radar Symposium IRS'07, Sep. 2007.

[2] F. Bordoni, M. Younis, E. M. Varona, N. Gebert, and G. Krieger, "Performance investigation on scan-on-receive and adaptive digital beam-forming for high-resolution wide-swath synthetic aperture radar," in Proceedings International ITG Workshop of Smart Antennas, Berlin, Germany., Feb. 2009.

[3] C. Schaefer, M. Younis, and M. Ludwig, "Advanced sar instrument based on digital beam forming," in Proceedings Advanced RF Sensors and Remote Sensing Instruments (ARSI), Noordwijk, The Netherlands, Nov. 2009.
[4] A. Moreira, I. Hajnsek, G. Krieger, K. Papathanassiou, M. Eineder, F. De Zan, M. Younis, and M. Werner, "Tandem-L: Monitoring the earth's dynamics with insar and pol-insar," in Proceedings International Workshop on Applications of Polarimetry and Polarimetric Interferometry (Pol-InSAR), Frascati, Italy, Jan. 2009.

[5] A. Freeman, G. Krieger, P. Rosen, M. Younis, W. Johnson, S. Huber, R. Jordan, and A. Moreira, "SweepSAR: Beamforming on receive using a reflector-phased array feed combination for spaceborne SAR," in Proceedings IEEE Radar Conference (RadarCon'09), Pasadena, U.S.A., May 2009.

[6] G. Krieger, N. Gebert, M. Younis, and A. Moreira, "Advanced synthetic aperture radar based on digital beamforming and waveform diversity," in Proc. IEEE Radar Conference (RadarCon), Rom, Italy, May 2008.

[7] (2009, Oct.) Introducing terrestar's integrated satelliteterrestrial system. TerreStar Corporation. [Online]. Available: http://www.terrestar.com/technology.php

[8] M. Younis, S. Huber, A. Patyuchenko, F. Bordoni, and G. Krieger, "Performance comparison of reflector- and planar-antenna based digital beamforming SAR," Int. Journal of Antennas and Propagation, vol. 2009, Jun. 2009. [Online]. Available: http://www.hindawi.com/journals/ijap/2009

[9] A. Patyuchenko, M. Younis, S. Huber, and G. Krieger, "Optimization aspects of the reflector antenna for the digital beam-forming SAR system," in Proc. European Conference on Synthetic Aperture Radar EUSAR'2010 (accepted), 2010.

[10] S. Huber, M. Younis, A. Patyuchenko, and G. Krieger, "Digital beam forming techniques for spaceborne reflector SAR systems," in Proc. European Conference on Synthetic Aperture Radar EUSAR'2010 (accepted), 2010.

[11] N. Gebert and G. Krieger, "Ultra-wide swath SAR imaging with continuous PRF variation," in Proc. European Conference on Synthetic Aperture Radar EUSAR'2010 (accepted), 2010.

[12] J. H. Blythe, "Radar systems," United States Patent Patent 4253098 , Feb., 1981.

[13] J. T. Kare, "Moving receive beam method and apparatus for synthetic aperture radar," United States Patent Patent 6175326 , Jan., 2001.

[14] M. Suess and W. Wiesbeck, "Side-looking synthetic aperture radar system," European Patent Patent EP 1241 487, Sep., 2002. 